IJMMS 29:11 (2002) 665-674

PII. S0161171202006014

http://ijmms.hindawi.com

(c) Hindawi Publishing Corp.

\title{
BREAKING THE SYMMETRY OF A CIRCULAR SYSTEM OF COUPLED HARMONIC OSCILLATORS
}

\author{
J. N. BOYD, R. G. HUDEPOHL, and P. N. RAYCHOWDHURY
}

Received 23 October 2000

\begin{abstract}
First we compute the natural frequencies of vibration of four identical particles coupled by ideal, massless harmonic springs. The four particles are constrained to move on a fixed circle. The initial computations are simplified by a transformation to symmetry coordinates. Then the symmetry of the vibrating system is broken by changing the mass of a single particle by a very small amount. We observe the effect of applying the symmetry transformation to the now slightly nonsymmetric system. We compute the new frequencies and compare them with the frequencies of the original symmetric system of oscillators. Results of similar calculations for 2, 3, 5, and 6 particles are given.
\end{abstract}

2000 Mathematics Subject Classification: 20C35.

1. Introduction. We have found the application of the representation theory of finite groups to the study of symmetrically coupled harmonic oscillators to be a fertile source of problems [11, 12]. We began by constructing unitary transformations from the irreducible matrix representations of the symmetry groups of the systems of oscillators. We then computed the natural frequencies of vibration for several complicated systems of oscillators after simplifying their equations of motion by means of the appropriate unitary transformations of their coordinates and velocities [1, 2, 3, 10]. We have also applied these transformations to the circuit equations for coupled L-C oscillators [4, 6]. In addition, we have looked at wave propagation in a two-dimensional lattice of springs and point masses [5].

We recognized that statistical sample variance as a quadratic polynomial form possesses the same symmetry as that of the total elastic potential energy of coupled harmonic oscillators forced to move on a fixed circle. We then used symmetry transformations to elaborate upon the geometrical interpretation of variance [7, 8]. Thereafter, we described a vibrating mechanical system to model within and between set variances [9].

In this paper, we return to these calculations which have for so long held our interest. We wondered what would happen if we broke the symmetry of a vibrating system and then applied to the new system the transformation derived from the original symmetry group. We performed the requisite calculations with the aid of Mathematica for several special cases in which the departures from symmetry were slight. The results seem quite interesting. 


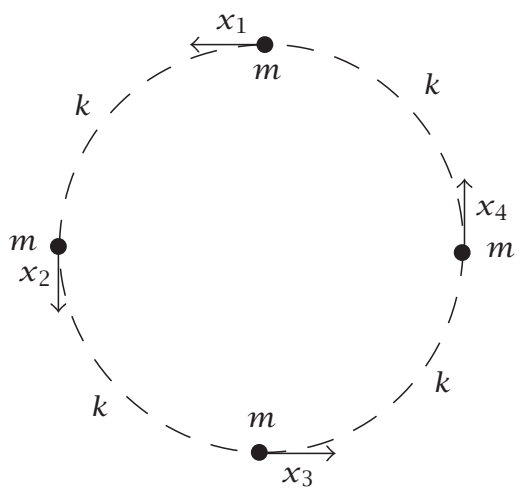

FIGURE 2.1. Four particles on a circle.

2. Motion on a circle. Our symmetric system consists of $n$ identical particles uniformly spaced around a fixed circle. Each particle is connected to its two nearest neighbors by identical Hooke's law springs which take the shape of the arc between adjacent particles. Each particle possesses mass $m$ while the springs are massless with force constant $\beta$. All motions of the system are confined to the circle. We will discover the natural frequencies for the vibrations of such a system in the case when $n=4$. In so doing, we will model all of the calculations which are required in the general case for arbitrary $n$ [1]. The geometry of the four particles interconnected with springs is indicated in Figure 2.1.

We denote the displacement of the $j$ th particle from its equilibrium position by $x_{j}$ and the velocity of the particle by $\dot{x}_{j}$. The total kinetic energy for the four particles may be written in matrix form as

$$
T=\left(\frac{m}{2}\right) \dot{X} I \operatorname{col}(\dot{X})
$$

where $I$ represents the $4 \times 4$ identity matrix, $\dot{X}=\left(\dot{x}_{1} \dot{x}_{2} \dot{x}_{3} \dot{x}_{4}\right)$, and $\operatorname{col}(\dot{X})$ is the transpose of the row velocity vector $\dot{X}$.

Suppose that the $j$ th and $k$ th particles are adjacent. Then the elastic potential energy, stored in the spring connecting the two particles, is $(\beta / 2)\left(x_{j}-x_{k}\right)^{2}$. The total elastic potential energy may be written as

$$
V=\left(\frac{\beta}{2}\right) X\left(\begin{array}{rrrr}
2 & -1 & 0 & -1 \\
-1 & 2 & -1 & 0 \\
0 & -1 & 2 & -1 \\
-1 & 0 & -1 & 2
\end{array}\right) \operatorname{col}(X)
$$

where $X=\left(x_{1} x_{2} x_{3} x_{4}\right)$ and $\operatorname{col}(X)$ is the transpose of $X$. 
The symmetry group for the system of coupled simple harmonic oscillators is the cyclic group of rotations of the circle by $90^{\circ}, 180^{\circ}, 270^{\circ}$, and $360^{\circ}$. The rotation by $360^{\circ}$ serves as the identity element in the group. The group of four complex fourth roots of unity $\{i,-1,-i, 1\}$ under complex multiplication is isomorphic to the rotation group. From these complex fourth roots of unity, we construct the unitary matrix

$$
U=\left(\frac{1}{2}\right)\left(\begin{array}{rrrr}
i & -1 & -i & 1 \\
-1 & 1 & -1 & 1 \\
-i & -1 & i & 1 \\
1 & 1 & 1 & 1
\end{array}\right)
$$

The entries in the first column of the matrix $U$ are the four different fourth roots of unity and the $j, k$ th entry in the matrix is $\left(u_{j 1}\right)^{k}[11]$.

Next, we express the kinetic and potential energies with respect to the complex symmetry coordinates $\left(z_{1}, z_{2}, z_{3}, z_{4}\right)$ and their conjugates $\left(\bar{z}_{1}, \bar{z}_{2}, \bar{z}_{3}, \bar{z}_{4}\right)$ together with the associated velocities $\left(\dot{z}_{1}, \dot{z}_{2}, \dot{z}_{3}, \dot{z}_{4}\right)$ and their conjugates $\left((\dot{\bar{z}})_{1},(\dot{\bar{z}})_{2},(\dot{\bar{z}})_{3},(\dot{\bar{z}})_{4}\right)$. We accomplish the transformations by means of matrix manipulations in the following manner:

$$
\begin{aligned}
T & =\left(\frac{m}{2}\right) \dot{X} U^{-1} U I U^{-1} U \operatorname{col}(\dot{X})=\left(\frac{m}{2}\right) \dot{\bar{Z}} I \operatorname{col}(\dot{Z}), \\
V & =\left(\frac{\beta}{2}\right) X U^{-1} U\left(\begin{array}{rrrr}
2 & -1 & 0 & -1 \\
-1 & 2 & -1 & 0 \\
0 & -1 & 2 & -1 \\
-1 & 0 & -1 & 2
\end{array}\right) U^{-1} U \operatorname{col}(X) \\
& =\left(\frac{\beta}{2}\right) \bar{Z}\left(\begin{array}{llll}
2 & 0 & 0 & 0 \\
0 & 4 & 0 & 0 \\
0 & 0 & 2 & 0 \\
0 & 0 & 0 & 0
\end{array}\right) \operatorname{col}(Z) .
\end{aligned}
$$

In this notation, $\bar{Z}=\left(\bar{z}_{1} \bar{z}_{2} \bar{z}_{3} \bar{z}_{4}\right), \operatorname{col}(Z)$ is the column symmetry displacement vector, $\dot{\bar{Z}}=\left(\dot{\bar{z}}_{1} \dot{\bar{z}}_{2} \dot{\bar{z}}_{3} \dot{\bar{z}}_{4}\right)$, and $\operatorname{col}(\dot{Z})$ is the column velocity vector.

The Lagrangian becomes

$$
L=T-V=\left(\frac{m}{2}\right)\left(\dot{\bar{z}}_{1} \dot{z}_{1}+\dot{\bar{z}}_{2} \dot{z}_{2}+\dot{\bar{z}}_{3} \dot{z}_{3}+\dot{\bar{z}}_{4} \dot{z}_{4}\right)-\left(\frac{\beta}{2}\right)\left(2 \bar{z}_{1} z_{1}+4 \bar{z}_{2} z_{2}+2 \bar{z}_{3} z_{3}\right)
$$

From $L$ we obtain the equations of motion as

$$
\frac{d}{d t}\left(\frac{\partial L}{\partial \dot{\bar{z}}_{j}}\right)-\frac{\partial L}{\partial \bar{z}_{j}}=0,
$$




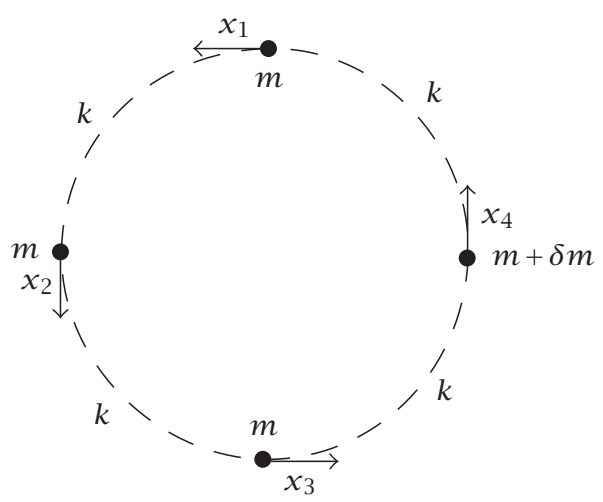

FIGURE 3.1. Four particles with their mass symmetry broken.

for $j=1,2,3,4$. The equations of motion are

$$
\begin{aligned}
m \ddot{z}_{1}+2 \beta z_{1} & =0, \\
m \ddot{z}_{2}+4 \beta z_{2} & =0, \\
m \ddot{z}_{3}+2 \beta z_{3} & =0, \\
m \ddot{z}_{4} & =0 .
\end{aligned}
$$

It is immediately clear that the frequencies of vibration for the complex symmetry coordinates are $f_{1}=(1 / 2 \pi) \sqrt{2 \beta / m}, f_{2}=(1 / \pi) \sqrt{\beta / m}, f_{3}=(1 / 2 \pi) \sqrt{2 \beta / m}$, and $f_{4}=0$. Since the transformations of coordinates and velocities leave the eigenvalues of the kinetic and potential energy matrices invariant, these are the natural frequencies of the system.

3. Breaking the symmetry. Now let the mass of a single particle be changed from $m$ to $m+\delta m$ with $|\delta m|$ very small when compared to $m$. All other masses and all springs remain as they were under the conditions of full symmetry. It does not matter which particle has been altered. So, for convenience, we will take it to be the $n$th particle with displacement and velocity $x_{n}$ and $\dot{x}_{n}$, respectively, which has been changed. We will demonstrate the effects of the broken circular symmetry in the case that $n=4$. The geometry of the new vibrating system is suggested by Figure 3.1.

The new kinetic energy may be written in matrix form as

$$
T=\left(\frac{m}{2}\right) \dot{X} I \operatorname{col}(\dot{X})+\left(\frac{\delta m}{2}\right) \dot{X}\left(\begin{array}{cccc}
0 & 0 & 0 & 0 \\
0 & 0 & 0 & 0 \\
0 & 0 & 0 & 0 \\
0 & 0 & 0 & 1
\end{array}\right) \operatorname{col}(\dot{X})
$$

while the potential energy matrix remains unchanged as given by (2.2). 
The vibrating system of particles and springs no longer possesses the symmetry of the rotation four-group, but it does not miss its former symmetry by far when $|\delta m| \ll m$. Our idea was to pursue the consequences of rewriting the Lagrangian by means of the symmetry transformations of (2.4) and (2.5).

The kinetic energy becomes

$$
\begin{aligned}
T & =\left(\frac{m}{2}\right) \dot{X} I \operatorname{col}(\dot{X})+\left(\frac{\delta m}{2}\right) \dot{X}\left(\begin{array}{cccc}
0 & 0 & 0 & 0 \\
0 & 0 & 0 & 0 \\
0 & 0 & 0 & 0 \\
0 & 0 & 0 & 1
\end{array}\right) \operatorname{col}(\dot{X}) \\
& =\left(\frac{m}{2}\right) \dot{X} U^{-1} U I U^{-1} U \operatorname{col}(\dot{X})+\left(\frac{\delta m}{2}\right) \dot{X} U^{-1} U\left(\begin{array}{cccc}
0 & 0 & 0 & 0 \\
0 & 0 & 0 & 0 \\
0 & 0 & 0 & 0 \\
0 & 0 & 0 & 1
\end{array}\right) U^{-1} U \operatorname{col}(\dot{X}) \\
& =\left(\frac{m}{2}\right) \dot{\bar{Z}} I \operatorname{col}(\dot{Z})+\left(\frac{\delta m}{8}\right) \dot{\bar{Z}}\left(\begin{array}{cccc}
1 & 1 & 1 & 1 \\
1 & 1 & 1 & 1 \\
1 & 1 & 1 & 1 \\
1 & 1 & 1 & 1
\end{array}\right) \operatorname{col}(\dot{Z}) .
\end{aligned}
$$

Since the symmetry of the connecting springs remains unchanged, the potential energy matrix retains the same form under transformation as that given before the symmetry was broken. The Lagrangian becomes $L=T-V$ where $T$ and $V$ are as given by (3.2) and (2.5), respectively. We have gained no computational advantage from the transformation, but we have obtained what we think is an interesting result. Breaking the symmetry of the original system by changing the mass of a single particle couples all of the velocities associated with the old symmetry coordinates in such a way that the new kinetic energy is shared equally among the old symmetry coordinates. The mathematics is quite straightforward and holds for all values of $n \in\{1,2,3, \ldots\}$.

4. The new frequencies. While an interesting exercise, the forcing of a rotational symmetry transformation upon the system of particles of masses $m, m$, and $m+\delta m$ complicated the actual problem of finding the new natural frequencies of the system after one mass had been changed. However, the system still possesses the symmetry of reflection across the line through the equilibrium positions of the second and fourth particles as shown in Figure 4.1.

We make use of the remaining symmetry to transform the original energies with the orthogonal reflection matrix

$$
W=\left(\frac{1}{\sqrt{2}}\right)\left(\begin{array}{rrrr}
-1 & 0 & 1 & 0 \\
0 & -1 & 0 & 1 \\
1 & 0 & 1 & 0 \\
0 & 1 & 0 & 1
\end{array}\right)
$$




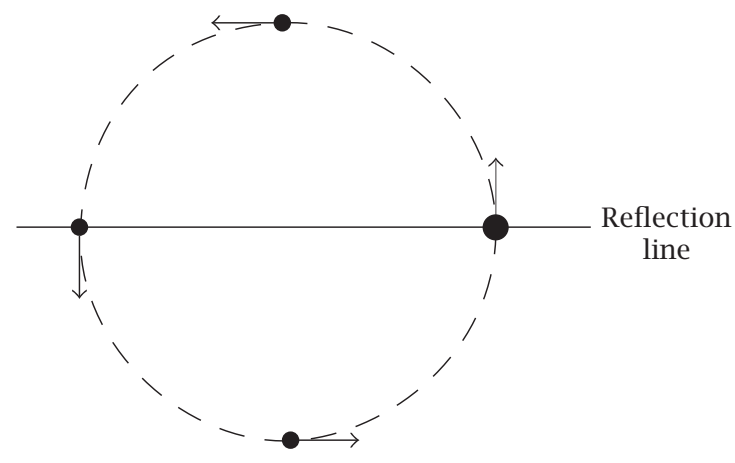

FIGURE 4.1. The four particles with reflection symmetry.

The kinetic energy should now be expressed as

$$
\begin{aligned}
T & =\left(\frac{m}{2}\right) \dot{X} I \operatorname{col}(\dot{X})+\left(\frac{\delta m}{2}\right) \dot{X}\left(\begin{array}{cccc}
0 & 0 & 0 & 0 \\
0 & 0 & 0 & 0 \\
0 & 0 & 0 & 0 \\
0 & 0 & 0 & 1
\end{array}\right) \operatorname{col}(\dot{X}) \\
& =\left(\frac{m}{2}\right) \dot{X} W^{-1} W I W^{-1} W \operatorname{col}(\dot{X})+\left(\frac{\delta m}{2}\right) \dot{X} W^{-1} W\left(\begin{array}{cccc}
0 & 0 & 0 & 0 \\
0 & 0 & 0 & 0 \\
0 & 0 & 0 & 0 \\
0 & 0 & 0 & 1
\end{array}\right) W^{-1} W \operatorname{col}(\dot{X}) \\
& =\left(\frac{m}{2}\right) \dot{Y} I \operatorname{col}(\dot{Y})+\left(\frac{\delta m}{2}\right) \dot{Y}\left(\begin{array}{cccc}
0 & 0 & 0 & 0 \\
0 & \frac{1}{2} & 0 & \frac{1}{2} \\
0 & 0 & 0 & 0 \\
0 & \frac{1}{2} & 0 & \frac{1}{2}
\end{array}\right) \operatorname{col}(\dot{Y}),
\end{aligned}
$$

where $\dot{Y}$ is the row velocity vector corresponding to the real symmetry row displacement vector $Y=\left(y_{1} y_{2} y_{3} y_{4}\right)$. The elastic potential energy becomes

$$
\begin{aligned}
V & =\left(\frac{\beta}{2}\right) X W^{-1} W\left(\begin{array}{rrrr}
2 & -1 & 0 & -1 \\
-1 & 2 & -1 & 0 \\
0 & -1 & 2 & -1 \\
-1 & 0 & -1 & 2
\end{array}\right) W^{-1} W \operatorname{col}(X) \\
& =\left(\frac{\beta}{2}\right) Y\left(\begin{array}{cccc}
2 & 0 & 0 & 0 \\
0 & 2 & 0 & 0 \\
0 & 0 & 2 & -2 \\
0 & 0 & -2 & 2
\end{array}\right) \operatorname{col}(Y) .
\end{aligned}
$$


From the Lagrangian

$$
L=T-V=\left(\frac{m}{2}\right)\left(\dot{y}_{1}^{2}+\dot{y}_{2}^{2}+\dot{y}_{3}^{2}+\dot{y}_{4}^{2}\right)+\frac{\delta m}{4}\left(\dot{y}_{2}+\dot{y}_{4}\right)^{2}-\beta\left(y_{1}^{2}+y_{2}^{2}+\left(y_{3}-y_{4}\right)^{2}\right)
$$

we derive the equations of motion as

$$
\frac{d}{d t}\left(\frac{\partial L}{\partial \dot{y}_{j}}\right)-\frac{\partial L}{\partial \dot{y}_{j}}=0 \quad \text { for } j=1,2,3,4
$$

the equations are

$$
\begin{gathered}
m \ddot{y}_{1}+2 \beta y_{1}=0, \\
\left(m+\frac{\delta m}{2}\right) \ddot{y}_{2}+\frac{\delta m}{2} \ddot{y}_{4}+2 \beta y_{2}=0, \\
m \ddot{y}_{3}+2 \beta\left(y_{3}-y_{4}\right)=0, \\
\frac{\delta m}{2} \ddot{y}_{2}+\left(m+\frac{\delta m}{2}\right) \ddot{y}_{4}-2 \beta\left(y_{3}-y_{4}\right)=0 .
\end{gathered}
$$

From the first of these four equations, we obtain the frequency $f_{1}^{*}=(1 / 2 \pi) \sqrt{2 \beta / m}$. To find the other frequencies, we turn to the three remaining equations and let $\ddot{y}_{j}=$ $-\omega^{2} y_{j}$ for $j=2,3,4$. We then write that

$$
\begin{gathered}
\left(-\omega^{2}\left(m+\frac{\delta m}{2}\right)+2 \beta\right) y_{2}-\omega^{2}\left(\frac{\delta m}{2}\right) y_{4}=0, \\
\left(-\omega^{2} m+2 \beta\right) y_{3}-2 \beta y_{4}=0 \\
-\omega^{2}\left(\frac{\delta m}{2}\right) y_{4}-2 \beta y_{3}+\left(-\omega^{2}\left(m+\frac{\delta m}{2}\right)+2 \beta\right) y_{4}=0 .
\end{gathered}
$$

These three equations have nontrivial solutions for $y_{2}, y_{3}$, and $y_{4}$ if and only if

$$
\operatorname{Det}\left(\begin{array}{ccc}
\left(-\omega^{2}\left(m+\frac{\delta m}{2}\right)+2 \beta\right) y_{2} & 0 & -\omega^{2}\left(\frac{\delta m}{2}\right) \\
0 & \left(-\omega^{2} m+2 \beta\right) & -2 \beta \\
-\omega^{2}\left(\frac{\delta m}{2}\right) & -2 \beta & -\omega^{2}\left(m+\frac{\delta m}{2}\right)+2 \beta
\end{array}\right)=0
$$

Expansion of this determinant yields a third degree polynomial equation in the variable $\omega^{2}$. One root of that equation is $\omega^{2}=0$. The other two solutions provided by Mathematica are complicated in appearance. The square roots of these solutions 
lead to the desired frequencies. The angular frequencies are

$$
\begin{aligned}
& \omega=\sqrt{\frac{3 m \beta+2 b \delta m+\beta \sqrt{m^{2}+2 m \delta m+2(\delta m)^{2}}}{m^{2}+m \delta m}}, \\
& \omega=\sqrt{\frac{3 m \beta+2 b \delta m+\beta \sqrt{m^{2}-2 m \delta m+2(\delta m)^{2}}}{m^{2}+m \delta}} .
\end{aligned}
$$

Since we are primarily interested in very small values of $\delta m$, we substitute for the expressions for $\omega$ the first two terms of their Maclaurin expansions in $\delta m$. Thus

$$
\begin{aligned}
& \omega \approx 2 \sqrt{\frac{\beta}{m} \sqrt{1-\frac{\delta m}{4 m}}} \approx 2 \sqrt{\frac{\beta}{m}}\left(1-\frac{\delta m}{8 m}\right), \\
& \omega \approx \sqrt{\frac{2 \beta}{m}} \sqrt{1-\frac{\delta m}{2 m}} \approx \sqrt{\frac{2 \beta}{m}}\left(1-\frac{\delta m}{4 m}\right) .
\end{aligned}
$$

It follows that

$$
\begin{aligned}
f_{2}^{*} & \approx \frac{1}{\pi} \sqrt{\frac{\beta}{m}} \sqrt{1-\frac{\delta m}{4 m}} \approx \frac{1}{\pi} \sqrt{\frac{\beta}{m}}\left(1-\frac{\delta m}{8 m}\right), \\
f_{3}^{*} & \approx \frac{1}{2 \pi} \sqrt{\frac{2 \beta}{m}} \sqrt{1-\frac{\delta m}{2 m}} \approx \frac{1}{2 \pi} \sqrt{\frac{2 \beta}{m}}\left(1-\frac{\delta m}{4 m}\right), \\
f_{4}^{*} & =0 .
\end{aligned}
$$

Recalling the frequencies $f_{1}, f_{2}, f_{3}$, and $f_{4}$ for the original four identical masses and springs, we see that

$$
f_{1}^{*}=f_{1}, \quad f_{2}^{*}=f_{2} \sqrt{1-\frac{\delta m}{4 m}}, \quad f_{3}^{*}=f_{3} \sqrt{1-\frac{\delta m}{2 m}}, \quad f_{4}^{*}=f_{4}=0
$$

OTHER RESUlts. We have also computed the frequencies for $n=2,3,5$, and 6 particles connected by springs on a fixed circle. In each case, the mass of single particle has been changed by a slight amount $\delta m$ as described in the previous section, and the computations leading to the results listed below are similar to those for $n=4$. We display the frequencies in Table 4.1.

The computations to obtain these frequencies are lengthy with length increasing as the number of particles $(n)$ increases. General results for arbitrary values of $n$ would seem to be difficult to obtain. However, the special examples which we have considered lead us to the hypothesis that breaking the circular symmetry by changing the mass $m$ of a single particle by a very small amount $\delta m$, either leaves a frequency unchanged or decreases the frequency by a factor of $1-\delta m /(n k m)$ where $k$ is a positive integer. 


\begin{tabular}{lll}
\hline$n$ & $\begin{array}{c}\text { Frequency } f \\
\text { (Full symmetry) }\end{array}$ & \multicolumn{1}{c}{$\begin{array}{c}\text { Frequency } f^{*} \\
\text { (Broken symmetry) }\end{array}$} \\
\hline 2 & $f_{1}=(1 / \pi) \sqrt{\beta / m}$ & $f_{1}^{*}=f_{1}(1-\delta m / 2(2 m))$ \\
& $f_{2}=0$ & $f_{2}^{*}=f_{2}=0$ \\
\hline 3 & $f_{1}=(1 / 2 \pi) \sqrt{3 \beta / m}$ & $f_{1}^{*}=f_{1}$ \\
& $f_{2}=(1 / 2 \pi) \sqrt{3 \beta / m}$ & $f_{2}^{*}=f_{2}(1-\delta m / 3 m)$ \\
& $f_{3}=0$ & $f_{3}^{*}=f_{3}=0$ \\
\hline & $f_{1}=(1 / 2 \pi) \sqrt{2 \beta / m}$ & $f_{1}^{*}=f_{1}$ \\
4 & $f_{2}=(1 / \pi) \sqrt{\beta / m}$ & $f_{2}^{*}=f_{2}(1-\delta m / 2(4 m))$ \\
& $f_{3}=(1 / 2 \pi) \sqrt{2 \beta / m}$ & $f_{3}^{*}=f_{3}(1-\delta m / 4 m)$ \\
& $f_{4}=0$ & $f_{4}^{*}=f_{4}=0$ \\
\hline 5 & $f_{1}=(1 / 2 \pi) \sqrt{\beta / m} \sqrt{(5-\sqrt{5}) / 2}$ & $f_{1}^{*}=f_{1}$ \\
& $f_{2}=(1 / 2 \pi) \sqrt{\beta / m} \sqrt{(5-\sqrt{5}) / 2}$ & $f_{2}^{*}=f_{2}(1-\delta m / 5 m)$ \\
& $f_{3}=(1 / 2 \pi) \sqrt{\beta / m} \sqrt{(5+\sqrt{5}) / 2}$ & $f_{3}^{*}=f_{3}$ \\
& $f_{4}=(1 / 2 \pi) \sqrt{\beta / m} \sqrt{(5+\sqrt{5}) / 2}$ & $f_{4}^{*}=f_{4}(1-\delta m / 5 m)$ \\
& $f_{5}=0$ & $f_{5}^{*}=f_{5}=0$ \\
\hline & $f_{1}=(1 / 2 \pi) \sqrt{\beta / m}$ & $f_{1}^{*}=f_{1}$ \\
$f_{2}=(1 / 2 \pi) \sqrt{\beta / m}$ & $f_{2}^{*}=f_{2}(1-\delta m / 6 m)$ \\
$f_{3}=(1 / 2 \pi) \sqrt{3 \beta / m}$ & $f_{3}^{*}=f_{3}$ \\
$f_{4}=(1 / 2 \pi) \sqrt{3 \beta / m}$ & $f_{4}^{*}=f_{4}(1-\delta m / 6 m)$ \\
$f_{5}=(1 / \pi) \sqrt{\beta / m}$ & $f_{5}^{*}=f_{5}(1-\delta m / 2(6 m))$ \\
$f_{6}=0$ & $f_{6}^{*}=f_{6}=0$ \\
\hline & &
\end{tabular}

\section{REFERENCES}

[1] J. N. Boyd and P. N. Raychowdhury, An application of projection operators to a onedimensional crystal, Bull. Inst. Math. Acad. Sinica 7 (1979), no. 2, 133-144.

[2] _ A one-dimensional crystal with nearest neighbors coupled through their velocities, Trans. ASME Ser. G J. Dynam. Systems Measurement and Control 103 (1981), 293296.

[3] _ Group representations in Lagrangian mechanics: an application to a twodimensional lattice, Phys. A 114 (1982), no. 1-3, 604-608.

[4] _ Finite group representation theory applied to coupled LC-circuits, J. Eng. Sci., King Saud Univ. 9 (1983), 43-49.

[5] _ Wave propagation in two-dimensional lattices, Pure Appl. Math. Sci. 20 (1984), $1-8$.

[6] _ A double chain of coupled circuits in analogy with mechanical lattices, Int. J. Math. Math. Sci. 14 (1991), no. 2, 403-406.

[7] _ A geometrical approach to maximizing a variance, Appl. Math. Modelling 18 (1994), 697-700.

[8]___ Computations for a vibrating system diagonalize the variance, Int. J. Math. Math. Sci. 18 (1995), no. 2, 411-415. 
[9] - Within and between set variances modelled with coupled harmonic oscillators, J. Theoret. Appl. Mech. 26 (1996), no. 1, 383-392.

[10] _ Lattice vibrations with Rayleigh dissipation, ANZIAM J. 42 (2000), no. 2, 244-253.

[11] M. Hamermesh, Group Theory and Its Application to Physical Problems, Addison-Wesley Series in Physics, Addison-Wesley, Massachusetts, 1962.

[12] A. Nussbaum, Group theory and normal modes, Amer. J. Phys. 36 (1968), 529-539.

J. N. BOYd, R. G. HudePohl, AND P. N. RAYCHOWDHURY: DEPARTMENT OF MATHEMATICAL SCIENCES, VIRGINIA COMMONWEALTH UNIVERSITY, RichmOND, VA 23284-2014, USA 


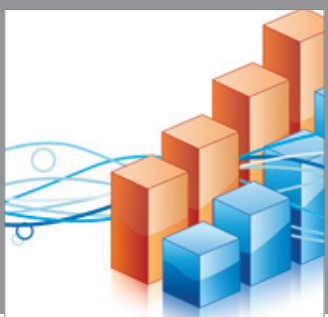

Advances in

Operations Research

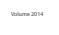

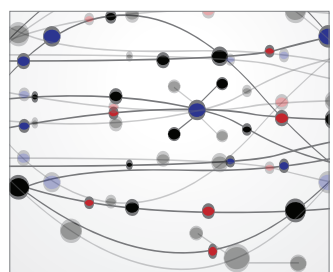

\section{The Scientific} World Journal
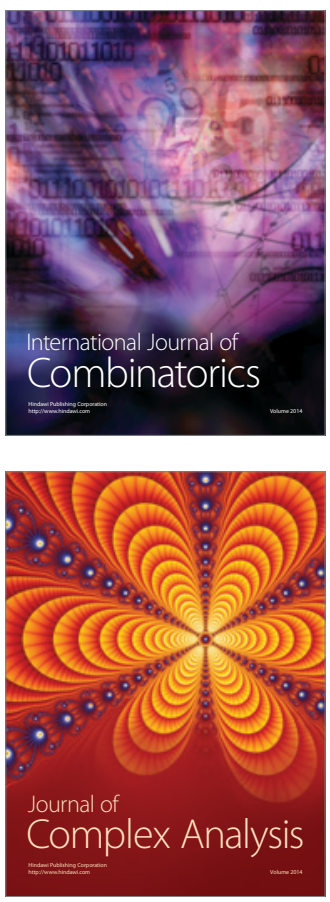

International Journal of

Mathematics and

Mathematical

Sciences
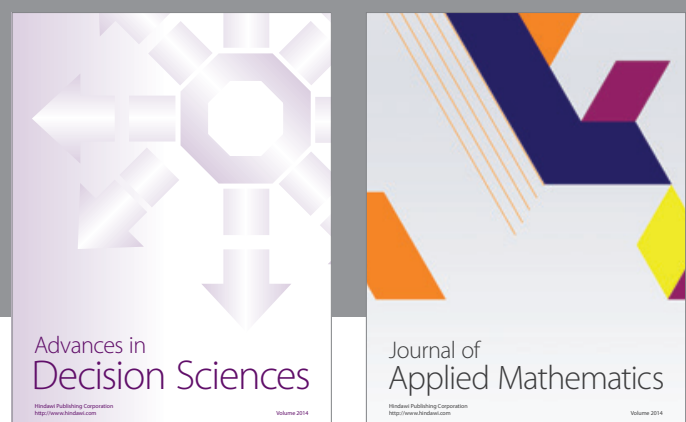

Journal of

Applied Mathematics
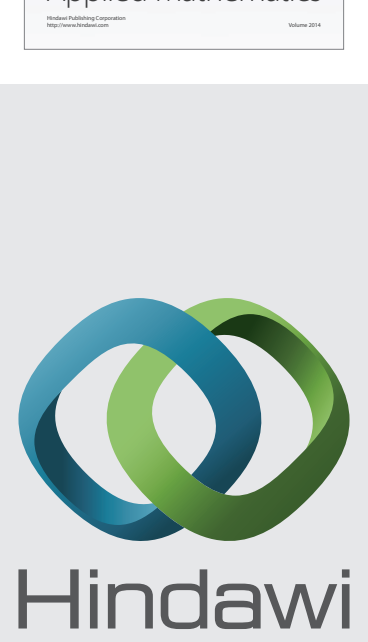

Submit your manuscripts at http://www.hindawi.com
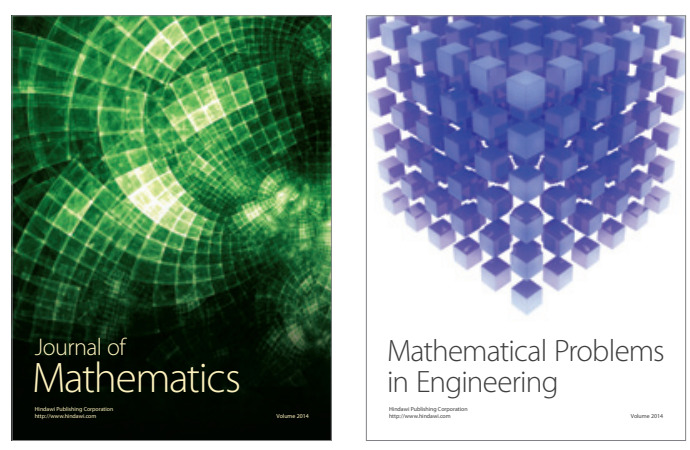

Mathematical Problems in Engineering
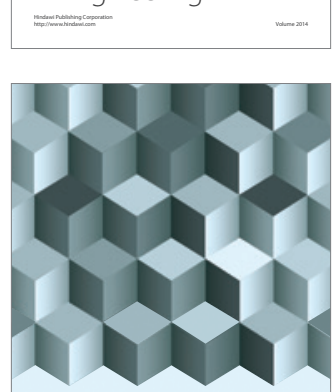

Journal of

Function Spaces
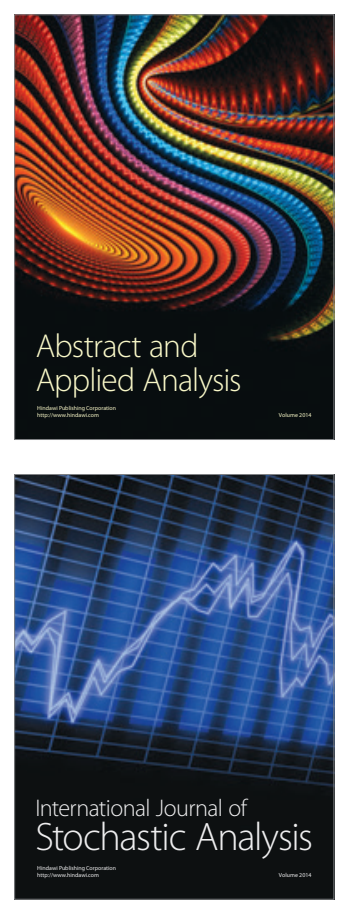

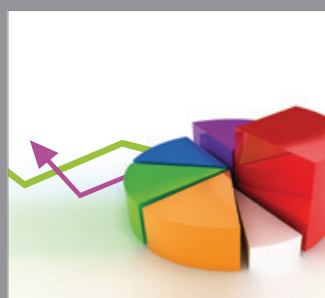

ournal of

Probability and Statistics

Promensencen
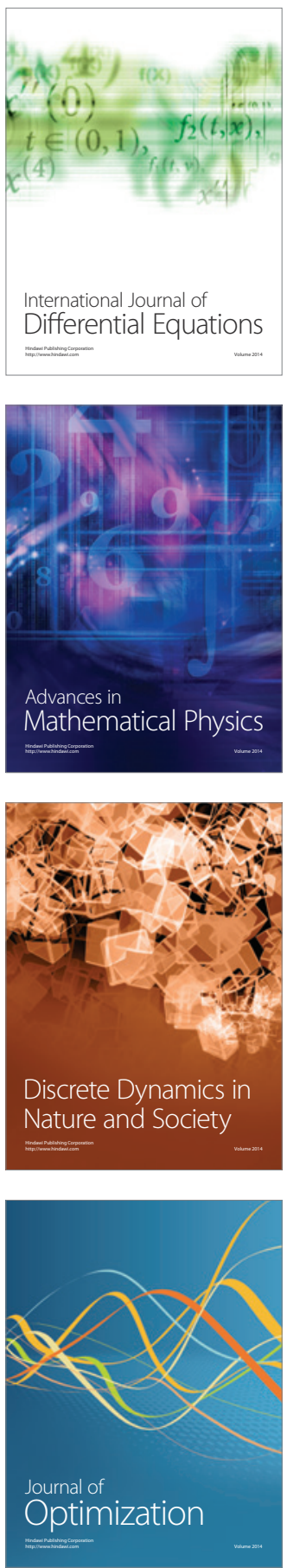